\title{
Entanglement measures for nonconformal D-branes
}

\author{
Arindam Lala* \\ Instituto de Física, Pontificia Universidad Católica de Valparaíso, Casilla 4059, Valparaíso, Chile
}

(Received 28 August 2020; accepted 24 November 2020; published 28 December 2020)

\begin{abstract}
We study various entanglement measures associated with certain nonconformal field theories. We consider nonconformal D $p$-brane backgrounds, which are dual to these field theories, for our holographic analysis. Restricting our interests in $p=1,2$, 4, we explicitly compute properties of holographic entanglement entropy and the entanglement wedge cross section, $\mathrm{E}_{W}$, corresponding to two parallel strip shaped boundary subregions in these setups. We study low and high temperature behaviors of these quantities analytically as well as by using numerical methods. In all cases, the $\mathrm{E}_{W}$ decreases monotonically with temperature. We observe discontinuous jumps in $\mathrm{E}_{W}$, while the width of (as well as the separation between) the subregions reaches critical values in all the cases considered. However, the corresponding holographic mutual information $I_{M}$ continuously decreases to zero for the aforementioned configurations. We also notice that the conjectured inequality $E_{W} \geq I_{M} / 2$ still holds for nonconformal field theories as well. We analytically determine the critical separation between these subregions that triggers a phase transition in the holographic mutual information.
\end{abstract}

DOI: 10.1103/PhysRevD.102.126026

\section{INTRODUCTION}

The gauge/gravity duality [1-3] has enriched our understanding of concepts related to quantum information theory. Perhaps the most prominent of all these examples is the holographic computations of entanglement entropy of the dual boundary theory of interest, which is particularly useful in determining the entanglement entropy of pure states [4-8]. This holographic entanglement entropy (HEE) proposal presents an interpretation of the entanglement entropy of the boundary field theory in terms of a geometric quantity, namely, the minimal surface, which is extended into the bulk. However, this prescription can easily be extended to the case of more than one boundary intervals [9]. In this regard, a related quantity known as the holographic mutual information (HMI), $\mathrm{I}_{M}(A, B)$, between two disjoint boundary intervals $A$ and $B$ can be determined [9-12]. Interestingly, $I_{M}(A, B) \geq 0$, and it is UV finite. Moreover, it undergoes a phase transition at some critical separation between the two intervals, in which case, the two subsystems become disentangled $[9,11]$.

On the other hand, in order to determine the entanglement entropy of mixed states, an interesting measure known as the holographic entanglement wedge cross

*arindam.physics1@gmail.com; arindam.lala@pucv.cl

Published by the American Physical Society under the terms of the Creative Commons Attribution 4.0 International license. Further distribution of this work must maintain attribution to the author(s) and the published article's title, journal citation, and DOI. Funded by SCOAP ${ }^{3}$. section (EWCS), $\mathrm{E}_{W}$, is of much discussion in recent times $[13,14]$. It is defined to be proportional to the minimal area of the entanglement wedge constructed out of the two intervals $A$ and $B{ }^{1}$ This quantity is conjectured to be holographic dual to entanglement of purification (EoP) [15], which measures the correlation between $A$ and $B$ and, for pure states, reduces to entanglement entropy. ${ }^{2}$ Although this proposal still has not been fully understood, there are certain indirect tests that this conjecture has passed, such as the following inequality [15]:

$$
\mathrm{E}_{W} \geq \frac{\mathrm{I}_{M}(A, B)}{2}
$$

which has been proven explicitly using the holographic duality $[13,14]$. Very recently, based on the formalism developed in $[13,14]$, various properties of EWCS, along with HEE and HMI, have been studied in different holographic setups [19-24]. ${ }^{3}$ All these examples mostly deal with conformal boundary theories, in which cases, the

\footnotetext{
${ }^{1}$ The entanglement wedge is a bulk region whose boundary is given by $\partial W_{A B}=A \cup B \cup \Gamma_{A B}^{m}$. Here, $\Gamma_{A B}^{m}$ is the minimal surface associated with the union $A B$.

${ }^{2}$ It must be mentioned that the $\mathrm{E}_{W}$ has also been proposed to be the gravity dual of holographic entanglement negativity [16] and reflected entropy $[17,18]$.

${ }^{3}$ Another promising direction, which is extensively based on the entanglement wedge construction, is the study of time evolution of mixed state correlation measures after global as well as local quantum quenches; see, for example, [25-27]. However, we shall not touch upon this aspect in this article.
} 
inequality (1) holds explicitly. Moreover, both $\mathrm{E}_{W}$ and HMI decrease monotonically, and $\mathrm{E}_{W}$ is shown to undergo a discontinuous phase transition at high temperature. ${ }^{4}$

The purpose of the present paper is to extend the study of certain aspects of EWCS, HEE, and HMI to nonconformal field theories, which are dual to nonconformal $\mathrm{D} p$-branes [28-32]. This will eventually extend the formalism to the study of nonconformal field theories as well, which is presently absent in the literature. This, in turn, could enable us to explore properties of scale noninvariant field theories abundant in nature.

The holographic correspondence between nonconformal $D p$-branes and their dual field theories were studied in details in [28], where the (super)gravity description is controlled by an effective dimensionless coupling constant $g_{\text {eff }}$. However, in a more general setup, the 10-dimensional theory of [28] can be dimensionally reduced to obtain an effective $(p+2)$-dimensional theory described by Einstein-dilaton gravity [29-32]. Interestingly, the spacetime metric obtained in the later case is found to be conformal to that of $(p+2)$-dimensional anti de Sitter space $\left(\operatorname{AdS}_{p+2}\right) .^{5}$

In this paper, we study the EWCS of two disjoint boundary intervals in the shape of parallel strips each of width $\ell$ and length $L$. The low as well as high temperature behaviors of the EWCS are also studied. We restrict ourselves to the cases $p=1,2,4$. However, results corresponding to the conformal $p=3$ case are also provided for sake of completeness of the analyses. We also discuss the behaviors of the HEE corresponding to the thermal boundary field theory along the line of analysis of [12,37-39]. In our analysis, we observe that, in all cases, the EWCS scales with the area of the entangling region contrary to the volume scaling of the HEE. In addition, it monotonically decreases to zero value for large temperatures beyond a certain critical value of the separation $h$ between the two disjoint intervals at which they become disentangled. However, in all cases, the corresponding HMI are monotonically decreasing functions as the critical separation between the strips is approached. Moreover, we also check the validity of the above inequality (1) explicitly in these setups.

The paper is organized as follows. In section II, we give a brief account of the nonconformal gravity backgrounds. In section III, we provide the computation of the EWCS and HEE corresponding to the strip shaped regions. The low

\footnotetext{
${ }^{4}$ Note that, in [20], nonmonotonous behaviors of HMI and EWCS have been reported, however in a different holographic setup.

${ }^{5}$ Starting from [28], nonconformal brane plane wave backgrounds have also been constructed in $[33,34]$, which are shown to allow nontrivial hyperscaling violating backgrounds upon appropriate dimensional reduction. Also, aspects of holographic entanglement entropy have been studied within these theories; see, e.g., $[35,36]$.
}

and high temperature behaviors of these quantities are studied subsequently. The values of the critical distance of separation between the entangling regions are also determined by studying the corresponding HMI. We finally conclude in section IV.

\section{THE HOLOGRAPHIC SETUP}

In this paper, we study quantum information quantities for certain nonconformal field theories. In order to do so, we consider nonconformal $D p$-brane backgrounds dual to these field theories [28]. Here, we first mention the corresponding constructions provided in [28] and then very briefly review a more general dimensionally reduced model following $[29,30]$, which we subsequently consider in all our computations.

The background generated from $N$ coincident extremal $D p$-branes in the string frame can be written as [28]

$$
\begin{aligned}
\mathrm{d} s^{2}= & \alpha^{\prime}\left[\frac{U^{(7-p) / 2}}{g_{\mathrm{YM}} \sqrt{\mathrm{d}_{p} N}}\left(-\mathrm{d} t^{2}+\sum_{i=1}^{p} \mathrm{~d} x_{i}^{2}\right)+\frac{g_{\mathrm{YM}} \sqrt{\mathrm{d}_{p} N}}{U^{(7-p) / 2}} \mathrm{~d} U^{2}\right. \\
& \left.+g_{\mathrm{YM}} \sqrt{\mathrm{d}_{p} N} U^{(p-3) / 2} \mathrm{~d} \Omega_{8-p}^{2}\right] \\
e^{\phi}= & (2 \pi)^{2-p} g_{\mathrm{YM}}^{2}\left(\frac{g_{\mathrm{YM}}^{2} N \mathrm{~d}_{p}}{U^{7-p}}\right)^{\frac{3-p}{4}}
\end{aligned}
$$

where

$$
\mathrm{d}_{p}=2^{7-p} \pi^{\frac{9-3 p}{2}} \Gamma\left(\frac{7-p}{2}\right), \quad U=\frac{r}{\alpha^{\prime}}
$$

and the limits $g_{\mathrm{YM}}^{2} \sim g_{s} \alpha^{\frac{p-3}{2}}=$ fixed, $U=$ fixed, and $\alpha^{\prime} \rightarrow 0$ have been taken. On top of that, the validity of the supergravity description is controlled by the dimensionless coupling constant $g_{\mathrm{eff}}=g_{\mathrm{YM}}^{2} N U^{p-3}$. In the nonextremal limit, the above solution (2) can be expressed as

$$
\begin{aligned}
\mathrm{d} s^{2}= & \alpha^{\prime}\left[\frac{U^{(7-p) / 2}}{g_{\mathrm{YM}} \sqrt{\mathrm{d}_{p} N}}\left(-f(U) \mathrm{d} t^{2}+\sum_{i=1}^{p} \mathrm{~d} x_{i}^{2}\right)\right. \\
& \left.+\frac{g(U) g_{\mathrm{YM}} \sqrt{\mathrm{d}_{p} N}}{U^{(7-p) / 2}} \mathrm{~d} U^{2}+g_{\mathrm{YM}} \sqrt{\mathrm{d}_{p} N} U^{(p-3) / 2} \mathrm{~d} \Omega_{8-p}^{2}\right] \\
e^{\phi}= & (2 \pi)^{2-p} g_{\mathrm{YM}}^{2}\left(\frac{g_{\mathrm{YM}}^{2} N \mathrm{~d}_{p}}{U^{7-p}}\right)^{\frac{3-p}{4}}
\end{aligned}
$$

where

$$
f(U)=1-\frac{U_{0}^{7-p}}{U^{7-p}}, \quad g(U)=\frac{1}{f(U)} \approx 1+\frac{U_{0}^{7-p}}{U^{7-p}} .
$$

However, in a more general setup, we can perform an $S^{8-p}$ Kaluza-Klein compactification of the above string-frame metric (4) to $(p+2)$-dimensions. The 
effective theory (in the Einstein frame) is then characterized by the $(p+2)$-dimensional Einstein-Dilaton action [29-32],

$$
\begin{aligned}
S= & \frac{N^{2}}{16 \pi G_{\mathrm{N}}^{p+2}}\left[\int \mathrm{d}^{p+2} x \sqrt{-g}\left(\mathcal{R}-\frac{1}{2} \partial_{\mu} \Phi \partial^{\mu} \Phi+V(\Phi)\right)\right. \\
& \left.-2 \int \mathrm{d}^{p+1} x \sqrt{-\gamma} \mathcal{K}\right],
\end{aligned}
$$

where

$$
\begin{gathered}
V(\Phi)=\frac{1}{2}(9-p)(7-p) N^{-2 \lambda / p} e^{a \Phi}, \quad \Phi=\frac{2 \sqrt{2(9-p)}}{\sqrt{p}(7-p)} \phi \\
a=-\frac{\sqrt{2}(p-3)}{\sqrt{p(9-p)}}, \quad \lambda=\frac{2(p-3)}{(7-p)},
\end{gathered}
$$

and $\mathcal{K}, \gamma_{a b}$ are the extrinsic curvature and the induced boundary metric, respectively. Also in (6), $G_{\mathrm{N}}^{p+2}$ is the $(p+2)$-dimensional Newton's constant. The above theory (6) allows for black brane solutions given by [29-32]

$$
\begin{gathered}
\mathrm{d} s^{2}=\left(N e^{\phi}\right)^{\frac{22}{p}}\left[\frac{u^{2}}{\Delta^{2}}\left(-f(u) \mathrm{d} t^{2}+\sum_{i=1}^{p} \mathrm{~d} x_{i}^{2}\right)+\frac{\Delta^{2} \mathrm{~d} u^{2}}{u^{2} f(u)}\right] \\
f(u)=1-\left(\frac{u_{0}}{u}\right)^{\frac{2(7-p)}{(5-p)}} \\
e^{\phi}=\frac{1}{N}\left(g_{\mathrm{YM}}^{2} N\right)^{\frac{7-p}{2(5-p)}}\left(\frac{u}{\Delta}\right)^{\frac{(p-7)(p-3)}{2(p-5)}} \\
u_{0}^{2}=\frac{U_{0}^{5-p}}{\left(g_{\mathrm{YM}}^{2} N\right)}, \quad \Delta=\frac{2}{5-p} .
\end{gathered}
$$

Now, the Hawking temperature of the $D p$-brane can be computed by the usual method of analytical continuation of the metric (9a) to the Euclidean sector, $t \rightarrow i \tau$ [40]. The resulting expression for the temperature can be calculated as

$$
T=\frac{1}{4 \pi \Delta^{2}} \frac{2(p-7)}{(p-5) z_{0}}
$$

where the following change in coordinates $u \rightarrow 1 / z$ and $u_{0} \rightarrow 1 / z_{0}$ has been taken into account $[31,32]$.
On the other hand, the thermal entropy of the $D p$-branes can be computed as

$$
\begin{aligned}
S_{\mathrm{th}} & =\frac{1}{4 G_{\mathrm{N}}^{p+2}} \int_{-\ell / 2}^{\ell / 2} \mathrm{~d} x \int_{-L / 2}^{L / 2} \prod_{i=1}^{p-1} \mathrm{~d} x_{i}\left(g_{x x} \prod_{i=1}^{p-1} g_{x_{i} x_{i}}\right)^{\frac{1}{2}} \\
& =\frac{L^{p-1} \ell}{4 G_{\mathrm{N}}^{p+2}}\left(g_{\mathrm{YM}}^{2} N\right)^{\frac{p-3}{5-p}}\left(\frac{1}{z_{0} \Delta}\right)^{\frac{p-9}{p-5}} .
\end{aligned}
$$

\section{ENTANGLEMENT WEDGE CROSS SECTION (EWCS)}

Let us consider two long parallel strips each of width $\ell$ and separated by a distance $h$. They indeed represent two subregions $A$ and $B$ and are shown as dark black lines in Fig. 1. We choose the following specific symmetric (around $x=0$ ) configurations for the subsystems [19-21]:

$$
\begin{aligned}
& A=\frac{h}{2}<x<\ell+\frac{h}{2} ; \quad-\frac{L}{2}<x_{2}, x_{3}, \ldots, x_{p-1}<\frac{L}{2}, \\
& B=-\ell-\frac{h}{2}<x<-\frac{h}{2} ; \quad-\frac{L}{2}<x_{2}, x_{3} \ldots, x_{p-1}<\frac{L}{2} .
\end{aligned}
$$

Now, the minimal surface, $\Gamma_{A B}^{m}$, that separates the two subsystems $A$ and $B$ is given by the vertical constant $x$ surface at $x=0$, which is a spacelike slice. The induced metric on this slice can be written as

$$
\begin{aligned}
\mathrm{d} s_{\Gamma_{A B}^{m}}^{2} & =\left(N e^{\phi}\right)^{\frac{22}{p}}\left[\frac{1}{z^{2} \Delta^{2}} \sum_{i=1}^{p-1} \mathrm{~d} x_{i}^{2}+\frac{\Delta^{2} \mathrm{~d} z^{2}}{z^{2} f(z)}\right], \\
f(z) & =1-\left(\frac{z}{z_{0}}\right)^{\frac{2(7-p)}{(5-p)}} .
\end{aligned}
$$

Next, we use the general expression for the entanglement wedge cross section (EWCS) corresponding to the two subregions $A$ and $B$ given by $[13,14,19-21]$

$$
\mathrm{E}_{W}=\frac{\operatorname{Area}\left(\Gamma_{A B}^{m}\right)}{4 G_{\mathrm{N}}^{p+2}} .
$$

Using (12) and (13), we finally obtain ${ }^{6}$

$$
\begin{aligned}
\mathrm{E}_{W}= & \frac{L^{p-1}\left(g_{\mathrm{YM}}^{2} N\right)^{\frac{p-3}{5-p}}}{4 G_{\mathrm{N}}^{p+2} \Delta^{\frac{p-1}{5-p}}} \int_{z_{t}(h)}^{z_{t}(2 \ell+h)} \mathrm{d} z \frac{z^{\frac{9-p}{p-5}}}{\sqrt{f(z)}} \\
= & \frac{L^{p-1}\left(g_{\mathrm{YM}}^{2} N\right)^{\frac{p-3}{5-p}}}{4 G_{\mathrm{N}}^{p+2} \Delta^{\frac{p-1}{5-p}}} \sum_{n=0}^{\infty} \frac{\Gamma\left(n+\frac{1}{2}\right)(p-5)}{\sqrt{\pi}(4+2 n(p-7)) \Gamma(n+1)} \\
& \times\left(\frac{z_{t}(2 \ell+h)^{\delta}}{z_{0}^{n \alpha}}-\frac{z_{t}(h)^{\delta}}{z_{0}^{n \alpha}}\right),
\end{aligned}
$$

where we have denoted

\footnotetext{
${ }^{6}$ Notice that the integration in (14) can be evaluated exactly; however, we find it convenient to express it in the series form $[12,38]$.
} 


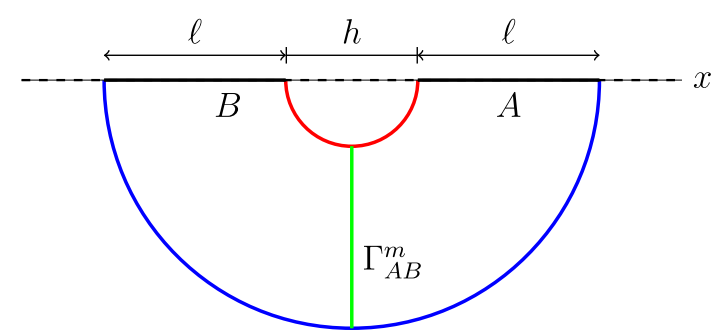

$-z_{0}$

FIG. 1. A schematic 2D diagram for computing entanglement wedge cross section (EWCS) $\Gamma_{A B}^{m}$. Here, we consider two parallel strips each of width $\ell$ separated by $h$. Here, $z_{0}$ represents the horizon of the brane.

$$
\alpha=\frac{2(7-p)}{(5-p)}, \quad \delta=\frac{4+2 n(p-7)}{(p-5)} .
$$

We now use the Ryu-Tagayanagi prescription [4,5] in order to find the HEE corresponding to a strip shaped region in the boundary of the geometry $(9 a)$, which can be expressed as [37]

$$
S_{\mathrm{EE}}=\frac{\mathcal{A}_{\gamma}}{4 G_{\mathrm{N}}^{p+2}},
$$

where $\mathcal{A}_{\gamma}$ is the area of the $p$-dimensional Ryu-Takayanagi surface $\gamma$.

In our analysis, we choose the strip parametrized as

$$
-\frac{\ell}{2} \leq x \leq \frac{\ell}{2}, \quad-\frac{L}{2} \leq x_{2}, \ldots, x_{p-1} \leq \frac{L}{2} .
$$

Thus, the HEE functional can be written as

$$
S_{\mathrm{EE}}=\frac{L^{p-1}}{2 G_{\mathrm{N}}^{p+2}}\left(g_{\mathrm{YM}}^{2} N\right)^{\frac{p-3}{5-p}} \Delta^{\frac{9-p}{p-5}} \int \mathrm{d} z z^{\frac{9-p}{p-5}} \sqrt{x^{\prime 2}+\frac{\Delta^{4}}{f(z)}} .
$$

Interestingly, the above functional (18) has no explicit dependence on $x(z)$, and hence, we can find the following first integral of motion by applying the conservation of energy of the system,

$$
x^{\prime}(z)= \pm \frac{\Delta^{2}}{\sqrt{f(z)\left(\left(\frac{z}{z_{t}}\right)^{2 \frac{(9-p)}{(p-5)}}-1\right)}},
$$

where $z_{t}$ is the turning point of the minimal surface. Also note that, in deriving (19), we have used the boundary condition $\lim _{x \rightarrow \infty} z=z_{t}$.

In the next step, we integrate (19) to obtain a relation between strip width $\ell$ and the turning point $z_{t}$ as

$$
\ell=2 \Delta^{2} z_{t} \int_{0}^{1} \mathrm{~d} v \frac{v^{\frac{9-p}{5-p}}}{\sqrt{f(v)} \sqrt{1-v^{2\left(\frac{(9-p)}{(5-p)}\right.}}},
$$

where we have defined $v=\frac{z}{z_{t}}$ and considered the fact that $1 \leq p \leq 4$.

Substituting (19) into (18), we finally obtain

$$
S_{\mathrm{EE}}=\frac{L^{p-1}}{2 G_{\mathrm{N}}^{p+2}}\left(g_{\mathrm{YM}}^{2} N\right)^{\frac{p-3}{5-p}} \frac{\Delta^{\frac{p-1}{p-5}}}{z_{t}^{\frac{4}{5-p}}} \int_{0}^{1} \frac{\mathrm{d} v}{v^{\frac{9-p}{5-p}}} \frac{1}{\sqrt{f(v)\left(1-v^{2 \frac{(9-p)}{(5-p)}}\right.}} .
$$

The integrations appearing in the above equations (20) and (21) can be evaluated easily; hence, the width and the HEE can, respectively, be expressed as [12,38]

$\ell=\Delta^{2} z_{t} \sum_{n=0}^{\infty} \frac{\Gamma\left(n+\frac{1}{2}\right) \Gamma\left(\frac{(n+1)(p-7)}{p-9}\right)}{\Gamma(n+1) \Gamma\left(\frac{3}{2}+n+\frac{2(n+1)}{(p-9)}\right)} \frac{(p-5)}{(p-9)}\left(\frac{z_{t}}{z_{0}}\right)^{2 n \frac{(7-p)}{(5-p)}}$,

$$
\begin{aligned}
S_{\mathrm{EE}}= & S_{\mathrm{sin}}+\frac{L^{p-1}}{2 G_{\mathrm{N}}^{p+2}}\left(g_{\mathrm{YM}}^{2} N\right)^{\frac{p-3}{5-p}} \frac{\Delta^{\frac{p-1}{p-5}}}{z_{t}^{5-p}}\left[\frac{\sqrt{\pi}(p-5) \Gamma\left(\frac{2}{p-9}\right)}{2(p-9) \Gamma\left(\frac{p-5}{2(p-9)}\right)}\right. \\
& \left.+\sum_{n=1}^{\infty} \frac{\Gamma\left(n+\frac{1}{2}\right) \Gamma\left(n+\frac{2(n+1)}{p-9}\right)}{\Gamma(n+1) \Gamma\left(\frac{p-5+2 n(p-7)}{2(p-9)}\right)} \frac{(p-5)}{2(p-9)}\left(\frac{z_{t}}{z_{0}}\right)^{2 n}\right],
\end{aligned}
$$

where the singular part of the HEE can be written as

$$
S_{\mathrm{sin}}=\frac{L^{p-1}}{2 G_{\mathrm{N}}^{p+2}}\left(g_{\mathrm{YM}}^{2} N\right)^{\frac{p-3}{5-p}} \frac{\Delta^{\frac{p-1}{p-5}}}{\epsilon^{\frac{4}{5-p}}}\left(\frac{5-p}{4}\right) .
$$

Notice that, in (24), $\epsilon$ is the UV cutoff.

At this point of discussion, note that we can get the expressions for $E_{W}$ as well as $S_{\mathrm{EE}}$ corresponding to the conformal (D3-brane) case by substituting $p=3$ in (14) and (21), respectively. In the next sections, we also compute the corresponding expressions for D3-branes for completeness of the analysis, which indeed match with those that already exist in the literature; see, e.g., [5,9, 19-21,38,39]. In addition, we shall also study the corresponding quantities by plotting their behaviors numerically. In our numerical analysis, we shall set the prefactor $\frac{L^{p-1}}{4 G_{\mathrm{N}}^{p+2}} \frac{\left(g_{\mathrm{YM}}^{2} N\right)^{\frac{(p-3)}{(5-p)}}}{\Delta^{\frac{p-1}{5-p}}}=1$.

\section{A. Low and high temperature behaviors of the HEE}

In this section, we study both the low and high temperature behaviors of the HEE (23) in the backgrounds (9a). 
In order to achieve this analytically, we need to solve the turning point $z_{t}$ in terms of the subregion length $\ell$. It is evident from (22) that this procedure can only be applied in the low and high temperature limits.

The low temperature limit is geometrically realized when the turning point $z_{t}$ corresponding to the RT surface(s) lies far away from the horizon $z_{0}$ of the black brane in the deep interior of the bulk: $z_{t} \ll z_{0}$. On the other hand, for the considered range of values of $p, 1 \leq p \leq 4$, the exponent $\frac{(7-p)}{(5-p)} \leq 3$. Hence, it is sufficient to keep terms up to order $\mathcal{O}\left(4 \frac{(7-p)}{(5-p)}\right)$ in the perturbative expansion. Thus, the resulting expression for the turning point in (22) may be written as

$$
\begin{aligned}
z_{t} \approx & \frac{\ell}{\Delta^{2} \Upsilon}\left[1-\frac{\sqrt{\pi}(p-5) \Gamma\left(\frac{2(p-7)}{p-9}\right)}{2(p-9) \Gamma\left(\frac{5}{2}+\frac{4}{p-9}\right)} \frac{1}{\Upsilon}\left(\frac{\ell}{\Delta^{2} z_{0} \Upsilon}\right)^{\frac{2(7-p)}{(5-p)}}\right. \\
& +\left\{\left(\frac{\sqrt{\pi}(p-5) \Gamma\left(\frac{2(p-7)}{p-9}\right)}{2(p-9) \Gamma\left(\frac{5}{2}+\frac{4}{p-9}\right)} \frac{1}{\Upsilon}\right)^{2}\right. \\
& \left.-\left(\frac{3 \sqrt{\pi}(p-5)}{8(p-9)} \frac{\Gamma\left(\frac{3(p-7)}{(p-9)}\right)}{\Gamma\left(\frac{7}{2}+\frac{6}{p-9}\right)} \frac{1}{\Upsilon}\right)\right\}\left(\frac{\ell}{\Delta^{2} z_{0} \Upsilon}\right)^{\frac{4(7-p)}{(7-p)}} \\
& \left.+\mathcal{O}\left(\frac{\ell}{\Delta^{2} z_{0} \Upsilon}\right)^{\frac{6(7-p)}{(5-p)}}\right],
\end{aligned}
$$

where we have defined

$$
\Upsilon=\frac{\sqrt{\pi}(p-5) \Gamma\left(\frac{p-7}{p-9}\right)}{(p-9) \Gamma\left(\frac{3}{2}+\frac{2}{p-9}\right)} .
$$

With this approximation and substituting (25) into (23), the final expressions for the HEE at low temperatures can be written as follows.

D1-brane

$$
\begin{aligned}
S_{\mathrm{EE}_{\mathrm{LT}}}^{(D 1)}= & \overline{\mathrm{C}}_{1}\left[S_{0}+\mathcal{C}_{1}\left(\frac{1}{\ell}\right)\left(1+\mathcal{C}_{2}\left(\frac{\pi T \ell}{3}\right)^{3}\right.\right. \\
& \left.\left.+\mathcal{C}_{3}\left(\frac{\pi T \ell}{3}\right)^{6}+\mathcal{O}(\pi T \ell / 3)^{7}\right)\right],
\end{aligned}
$$

where we have denoted

$$
\overline{\mathrm{C}}_{1}=\frac{\left(g_{\mathrm{YM}}^{2} N\right)^{-\frac{1}{2}}}{4 G_{\mathrm{N}}^{3}}, \quad S_{0}=\frac{2}{\epsilon},
$$

and the other constants $\mathcal{C}_{1}, \mathcal{C}_{2}$, and $\mathcal{C}_{3}$ are constants. We have explicitly written down the forms of these constants and those that appear below $\left(\mathcal{D}_{i}, \mathcal{B}_{i}\right.$, and $\left.\mathcal{E}_{i}, i=1,2,3\right)$ in appendix.

\footnotetext{
${ }^{7}$ Here and what follows, the subscripts LT and HT denote low temperature and high temperature, respectively.
}

D2-brane

$$
\begin{aligned}
S_{\mathrm{EE}_{\mathrm{LT}}}^{(D 2)}= & \overline{\mathrm{C}}_{2}\left[S_{0}+\mathcal{D}_{1}\left(\frac{1}{\ell}\right)^{4 / 3}\left(1+\mathcal{D}_{2}\left(\frac{8 \pi T \ell}{15}\right)^{10 / 3}\right.\right. \\
& \left.\left.+\mathcal{D}_{3}\left(\frac{8 \pi T \ell}{15}\right)^{20 / 3}+\mathcal{O}(8 \pi T \ell / 15)^{19 / 3}\right)\right]
\end{aligned}
$$

where

$$
\overline{\mathrm{C}_{2}}=\frac{L\left(g_{\mathrm{YM}}^{2} N\right)^{-\frac{1}{3}}}{4 G_{\mathrm{N}}^{4} \Delta^{\frac{1}{3}}}, \quad S_{0}=\left(\frac{3}{2}\right)\left(\frac{1}{\epsilon}\right)^{4 / 3},
$$

and $\mathcal{D}_{1}, \mathcal{D}_{2}$, and $\mathcal{D}_{3}$ are constants.

D3-brane [5,9,19-21,38,39]

$$
\begin{aligned}
S_{\mathrm{EE}_{\mathrm{LT}}}^{(D 3)}= & \overline{\mathrm{C}_{3}}\left[S_{0}+\mathcal{B}_{1}\left(\frac{1}{\ell}\right)^{2}\left(1+\mathcal{B}_{2}(\pi T \ell)^{4}\right.\right. \\
& \left.\left.+\mathcal{B}_{3}(\pi T \ell)^{8}+\mathcal{O}(\pi T \ell)^{10}\right)\right]
\end{aligned}
$$

where

$$
\overline{\mathrm{C}_{3}}=\frac{L^{2}}{4 G_{\mathrm{N}}^{5}}, \quad S_{0}=\frac{1}{\epsilon^{2}},
$$

and $\mathcal{B}_{1}, \mathcal{B}_{2}$, and $\mathcal{B}_{3}$ are constants.

\section{D4-brane}

$$
\begin{aligned}
S_{\mathrm{EE}_{\mathrm{LT}}}^{(D 4)}= & \overline{\mathrm{C}}_{4}\left[S_{0}+\mathcal{E}_{1}\left(\frac{1}{\ell}\right)^{4}\left(1+\mathcal{E}_{2}\left(\frac{8 \pi T \ell}{3}\right)^{6}\right.\right. \\
& \left.\left.+\mathcal{E}_{3}\left(\frac{8 \pi T \ell}{3}\right)^{12}+\mathcal{O}(8 \pi T \ell / 3)^{18}\right)\right],
\end{aligned}
$$

with

$$
\overline{\mathrm{C}}_{4}=\frac{L^{3}\left(g_{\mathrm{YM}}^{2} N\right)}{4 G_{\mathrm{N}}^{6} \Delta^{3}}, \quad S_{0}=\left(\frac{1}{2}\right)\left(\frac{1}{\epsilon}\right)^{4},
$$

and $\mathcal{E}_{1}, \mathcal{E}_{2}$, and $\mathcal{E}_{3}$ are constants.

Notice that when the temperature is zero $(T=0)$, the leading order finite terms appearing in (27), (29), (31), and (33) agree with those prescribed in [37].

On the other hand, at high temperatures, the turning point $z_{t}$ approaches the horizon of the black branes, $z_{t} \rightarrow z_{0}$, and indeed wraps a part of the horizon. Thus, the leading contribution comes from the near horizon part of the surface. On top of that, the entire bulk geometry contributes in the form of subleading terms $[12,38]$. The following combination is found to be converging as the limit $z_{t} \rightarrow z_{0}$ is taken, and hence, we proceed our analysis with this combination $[12,21,38]$. 


$$
\begin{aligned}
S_{\mathrm{EE}} & -\frac{L^{p-1}\left(g_{\mathrm{YM}}^{2} N\right)^{\frac{p-3}{5-p}}}{4 G_{\mathrm{N}}^{p+2}}\left(\frac{1}{\Delta z_{t}}\right)^{\frac{9-p}{5-p}} \ell \\
= & \frac{L^{p-1}\left(g_{\mathrm{YM}}^{2} N\right)^{\frac{p-3}{5-p}}}{2 G_{\mathrm{N}}^{p+2} \Delta^{\frac{p-1}{5-p}}}\left(\frac{1}{z_{t}}\right)^{\frac{4}{5-p}} \int_{\epsilon / z_{t}}^{1} \mathrm{~d} v \frac{\sqrt{1-v^{\frac{2(9-p)}{5-p}}}}{\sqrt{f(v)} v^{\frac{9-p}{5-p}}} .
\end{aligned}
$$

Using (35), we can now recast the finite part of the HEE as

$$
\begin{aligned}
S_{\mathrm{EE}}= & \frac{L^{p-1}\left(g_{\mathrm{YM}}^{2} N\right)^{\frac{p-3}{5-p}}}{4 G_{\mathrm{N}}^{p+2}}\left(\frac{1}{\Delta z_{t}}\right)^{\frac{9-p}{5-p}} \ell+\frac{L^{p-1}\left(g_{\mathrm{YM}}^{2} N\right)^{\frac{p-3}{5-p}}}{2 G_{\mathrm{N}}^{p+2} \Delta^{\frac{p-1}{5-p}}}\left(\frac{1}{z_{t}}\right)^{\frac{4}{5-p}} \\
& \times\left[\frac{(p-5)}{4}{ }_{2} F_{1}\left(\frac{1}{2}, \frac{2}{p-9} ; \frac{p-7}{p-9} ; 1\right)\right. \\
& \left.+\int_{0}^{1} \mathrm{~d} v\left(\frac{\sqrt{1-v^{\frac{2(9-p)}{5-p}}}}{\sqrt{f(v)} v^{\frac{9-p}{5-p}}}-\frac{1}{v^{\frac{9-p}{5-p}} \sqrt{1-v^{\frac{2(9-p)}{5-p}}}}\right)\right]
\end{aligned}
$$

where ${ }_{2} F_{1}(a, b ; c ; z)$ is the usual hypergeometric function.

Finally, considering the limit $z_{t} \rightarrow z_{0}$, we can read off the expression for the HEE at high temperatures from (36) as $[12,38]$

$$
\begin{aligned}
S_{\mathrm{EE}_{\mathrm{HT}}}= & S_{\mathrm{sin}}+\frac{V\left(g_{\mathrm{YM}}^{2} N\right)^{\frac{p-3}{5-p}}}{4 G_{\mathrm{N}}^{p+2} \Delta^{\frac{9-p}{5-p}}}\left(\frac{2 \pi \Delta^{2}(p-5)}{(p-7)} T\right)^{\frac{9-p}{5-p}} \\
& \times\left\{1+2\left(\frac{(p-7)}{2 \pi \ell T(p-5)}\right) \Theta\right\},
\end{aligned}
$$

where $S_{\text {sin }}$ is the singular part of the HEE given by (24), and

$$
\begin{aligned}
\Theta= & \frac{\sqrt{\pi}(p-5)}{(p-9)}\left[\frac{\Gamma\left(\frac{2}{p-9}\right)}{2 \Gamma\left(\frac{p-5}{2(p-9)}\right)}-\frac{\Gamma\left(\frac{p-7}{p-9}\right)}{\Gamma\left(\frac{3}{2}+\frac{2}{(p-9)}\right)}\right] \\
& +\sum_{n=1}^{\infty} \frac{(p-5) \Gamma\left(n+\frac{1}{2}\right)}{(p-9) \Gamma(n+1)}\left[\frac{\Gamma\left(n+\frac{2(n+1)}{p-9}\right)}{2 \Gamma\left(\frac{p-5+2 n(p-7)}{2(p-9)}\right)}\right. \\
& \left.-\frac{\Gamma\left(\frac{(n+1)(p-7)}{p-9}\right)}{\Gamma\left(\frac{3}{2}+n+\frac{2(n+1)}{(p-9)}\right)}\right] .
\end{aligned}
$$

Note that the finite term of the HEE in (37) scales as the volume of the entangling region $V=\ell L^{p-1}$. Also, this term is proportional to the thermal entropy (11). This is expected since at high temperature regime, the contributions to the HEE of the thermal boundary field theory come from the thermal fluctuations. Furthermore, when $p=3$, the HEE at high temperature, (37), corresponds to the conformal case as discussed in $[5,9,19-21,38,39]$.

\section{B. Low and high temperature behaviors of the EWCS}

In order to study the low and high temperature behaviors of the EWCS, we first notice that we can achieve this by taking into account the following three intrinsic scales associated with the theory: the separation distance $h$ between the two entangling regions, the width $\ell$ of each of the regions, and the temperature $T$ of the boundary theory $[12,19-21,38]$. Subsequently, in this case, the low temperature limit corresponds to $h T \ll \ell T \ll 1$. Note that this corresponds to considering the temperature smaller than both the length scales associated with $h$ and $\ell$. On the other hand, the high temperature limit may be defined by considering the following inequality $h T \ll 1 \ll \ell T$, in which case, the temperature is large compared to the scale associated with $\ell$ but small compared to that associated with $h{ }^{8}$ Interestingly, this high temperature limit further amounts to taking the following two approximations: $z_{t}(h) \ll z_{0}$ and $z_{t}(2 \ell+h) \rightarrow z_{0}$. The first approximation amounts to considering only the leading order term in the second term within the braces in (14), while in order for the second approximation to be valid, we must ensure the convergence of the first sum in (14). It is trivial to check that this is indeed the case. Thus, it is safe to consider the limit $z_{t}(2 \ell+h) \rightarrow z_{0}$.

Let us now discuss the different cases corresponding to $p=1,2,4$ separately, which correspond to D1-, D2-, and D4-branes, respectively. We have also provided the corresponding expressions for D3-branes (see, e.g., [19-21]) for completeness.

\section{Dl-brane}

In the low temperature limit $h T \ll \ell T \ll 1$, the EWCS (14) for the D1-branes can be computed as

$$
\begin{aligned}
\mathrm{E}_{W_{\mathrm{LT}}}^{(D 1)}= & \frac{\left(g_{\mathrm{YM}}^{2} N\right)^{-\frac{1}{2}}}{4 G_{\mathrm{N}}^{3}} \sum_{n=0}^{\infty} \frac{\Gamma\left(n+\frac{1}{2}\right)}{\sqrt{\pi} \Gamma(n+1)} \\
& \times\left(\frac{z_{t}(2 \ell+h)^{3 n-1}-z_{t}(h)^{3 n-1}}{3 n-1}\right) \cdot \frac{1}{z_{0}^{3 n}} \\
= & \frac{\left(g_{\mathrm{YM}}^{2} N\right)^{-\frac{1}{2}}}{4 G_{\mathrm{N}}^{3}}\left[\frac{\sqrt{\pi} \Gamma\left(\frac{3}{4}\right)}{8 \Gamma\left(\frac{5}{4}\right)}\left(\frac{1}{h}-\frac{1}{2 \ell+h}\right)\right. \\
& \left.+\frac{64 \Gamma\left(\frac{5}{4}\right)^{2}}{\pi \Gamma\left(\frac{3}{4}\right)^{2}}\left(1-\frac{\sqrt{\pi} \Gamma\left(\frac{5}{4}\right)}{\Gamma\left(\frac{3}{4}\right)}\right) \ell(\ell+h)\left(\frac{\pi T}{3}\right)^{3}+\cdots\right],
\end{aligned}
$$

where we have used (10) and (25).

On the other hand, considering the aforementioned high temperature limits, the behavior of the EWCS can be determined as

\footnotetext{
${ }^{8}$ In our calculations, we shall not take into account another limit $\ell T \ll h T$ or $1 \ll \ell T, 1 \ll h T$ as this corresponds to the disentangling phase of the two subregions [12,19,21,38,39].
} 


$$
\begin{aligned}
\mathrm{E}_{W_{\mathrm{HT}}}^{(D 1)} \approx & \frac{\left(g_{\mathrm{YM}}^{2} N\right)^{-\frac{1}{2}}}{4 G_{\mathrm{N}}^{3}} T\left[\frac{\pi \tilde{\mathcal{C}}_{1}}{3}+\frac{\sqrt{\pi} \Gamma\left(\frac{3}{4}\right)}{8 \Gamma\left(\frac{5}{4}\right)}\left(\frac{1}{h T}\right)\right. \\
& \left.+\frac{16 \pi^{\frac{5}{2}}}{27}\left(\frac{\Gamma\left(\frac{5}{4}\right)}{\Gamma\left(\frac{3}{4}\right)}\right)^{3}(h T)^{2}\right],
\end{aligned}
$$

where we have defined $\tilde{\mathcal{C}}_{1}=\sum_{n=0}^{\infty} \frac{\Gamma\left(n+\frac{1}{2}\right)}{\sqrt{\pi}(3 n-1) \Gamma(n+1)}$.

D2-brane

In a similar manner, the low temperature behavior of EWCS for the D2-branes (14) can be determined as

$$
\begin{aligned}
\mathrm{E}_{W_{\mathrm{LT}}}^{(D 2)}= & \frac{L\left(g_{\mathrm{YM}}^{2} N\right)^{-\frac{1}{3}}}{4 G_{\mathrm{N}}^{4} \Delta^{\frac{1}{3}}} \sum_{n=0}^{\infty} \frac{\Gamma\left(n+\frac{1}{2}\right)}{\sqrt{\pi} \Gamma(n+1)} \\
& \times\left(\frac{z_{t}(2 \ell+h)^{\frac{10 n-4}{3}}-z_{t}(h)^{\frac{10 n-4}{3}}}{10 n-4}\right) \cdot \frac{3}{z_{0}^{\frac{10}{3} n}} \\
= & \frac{L\left(g_{\mathrm{YM}}^{2} N\right)^{-\frac{1}{3}}}{4 G_{\mathrm{N}}^{4} \Delta^{\frac{1}{3}}}\left[\frac{3(2 \pi)^{\frac{2}{3}}}{(21)^{\frac{4}{3}}}\left(\frac{\Gamma\left(\frac{5}{7}\right)}{\Gamma\left(\frac{17}{14}\right)}\right)^{\frac{4}{3}}\left(\frac{1}{h^{\frac{4}{3}}}-\frac{1}{(2 \ell+h)^{\frac{4}{3}}}\right)\right. \\
& +\frac{441}{16 \pi} \frac{\Gamma\left(\frac{17}{14}\right)^{2}}{\Gamma\left(\frac{5}{7}\right)^{2}} \ell(\ell+h)\left(1-\frac{2 \Gamma\left(\frac{17}{14}\right) \Gamma\left(\frac{10}{7}\right)}{\Gamma\left(\frac{5}{7}\right) \Gamma\left(\frac{27}{14}\right)}\right) \\
& \left.\times\left(\frac{8 \pi T}{15}\right)^{\frac{10}{3}}+\cdots\right] .
\end{aligned}
$$

On the other hand, at high temperature, the corresponding EWCS behaves as

$$
\begin{aligned}
\mathrm{E}_{W_{\mathrm{HT}}}^{(D 2)} \approx & \frac{L\left(g_{\mathrm{YM}}^{2} N\right)^{-\frac{1}{3}}}{4 G_{\mathrm{N}}^{4} \Delta^{\frac{1}{3}}} T^{\frac{4}{3}}\left[\tilde{\mathcal{C}}_{2}\left(\frac{8 \pi}{15}\right)^{\frac{4}{3}}+\frac{3(2 \pi)^{\frac{2}{3}}}{21^{\frac{4}{3}}}\left(\frac{\Gamma\left(\frac{5}{7}\right)}{\Gamma\left(\frac{17}{14}\right)}\right)^{\frac{4}{3}}\left(\frac{1}{h T}\right)^{\frac{4}{3}}\right. \\
& \left.+\frac{441 \Gamma\left(\frac{17}{14}\right)^{3} \Gamma\left(\frac{10}{7}\right)}{32 \pi} \frac{8 \pi}{\Gamma\left(\frac{5}{7}\right)^{3} \Gamma\left(\frac{27}{14}\right)}\left(\frac{10}{15}\right)^{\frac{10}{3}}(h T)^{2}\right]
\end{aligned}
$$

with $\tilde{\mathcal{C}}_{2}=\sum_{n=0}^{\infty} \frac{3 \Gamma\left(n+\frac{1}{2}\right)}{\sqrt{\pi}(10 n-4) \Gamma(n+1)}$.

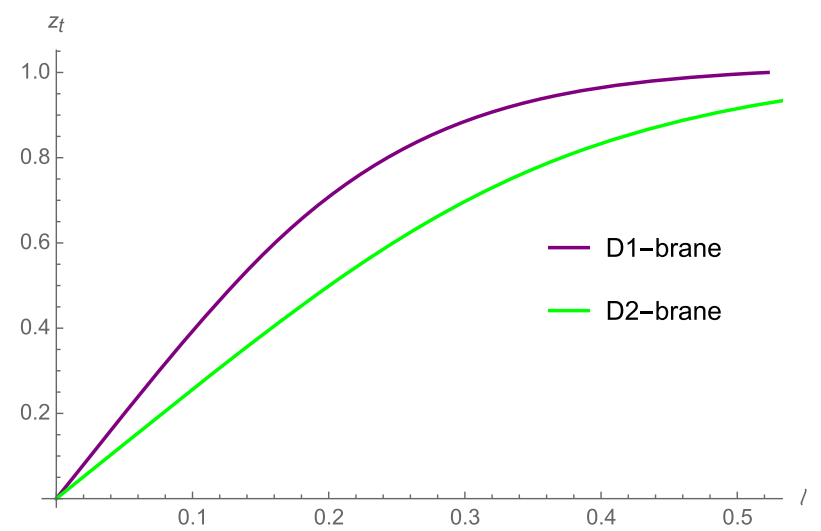

D3-brane [19-21]

The low temperature behavior of EWCS for the D3branes (14) can be expressed as

$$
\begin{aligned}
\mathrm{E}_{W_{\mathrm{LT}}}^{(D 3)}= & \frac{L^{2}}{4 G_{\mathrm{N}}^{5}} \sum_{n=0}^{\infty} \frac{\Gamma\left(n+\frac{1}{2}\right)}{2 \sqrt{\pi} \Gamma(n+1)} \\
& \times\left(\frac{z_{t}(2 \ell+h)^{2(2 n-1)}-z_{t}(h)^{2(2 n-1)}}{2 n-1}\right) \cdot \frac{1}{z_{0}^{4 n}} \\
= & \frac{L^{2}}{4 G_{\mathrm{N}}^{5}}\left[\frac{2 \pi \Gamma\left(\frac{2}{3}\right)^{2}}{\Gamma\left(\frac{1}{6}\right)^{2}}\left(\frac{1}{h^{2}}-\frac{1}{(2 \ell+h)^{2}}\right)\right. \\
& \left.+\frac{9 \Gamma\left(\frac{7}{6}\right)^{2}}{\pi \Gamma\left(\frac{2}{3}\right)^{2}} \ell(\ell+h)\left(1-\frac{2 \Gamma\left(\frac{7}{6}\right) \Gamma\left(\frac{4}{3}\right)}{\Gamma\left(\frac{2}{3}\right) \Gamma\left(\frac{11}{6}\right)}\right)(\pi T)^{4}+\cdots\right] .
\end{aligned}
$$

On the other hand, at high temperature, the corresponding EWCS behaves as

$$
\begin{aligned}
\mathrm{E}_{W_{\mathrm{HT}}}^{(D 3)} \approx & \frac{L^{2}}{4 G_{\mathrm{N}}^{5}} T^{2}\left[\tilde{\mathcal{C}}_{3} \pi^{2}+\frac{4 \pi \Gamma\left(\frac{2}{3}\right)^{2}}{\Gamma\left(\frac{1}{6}\right)^{2}}\left(\frac{1}{h T}\right)^{2}\right. \\
& \left.+\frac{9 \pi^{3} \Gamma\left(\frac{7}{6}\right)^{3} \Gamma\left(\frac{4}{3}\right)}{\Gamma\left(\frac{2}{3}\right)^{3} \Gamma\left(\frac{11}{6}\right)}(h T)^{2}\right]
\end{aligned}
$$

with $\tilde{\mathcal{C}}_{3}=\sum_{n=0}^{\infty} \frac{\Gamma\left(n+\frac{1}{2}\right)}{2 \sqrt{\pi}(2 n-1) \Gamma(n+1)}$.

\section{D4-brane}

Similar to the previous three cases, the low temperature behavior of EWCS (14) corresponding to the D4-brane is given by

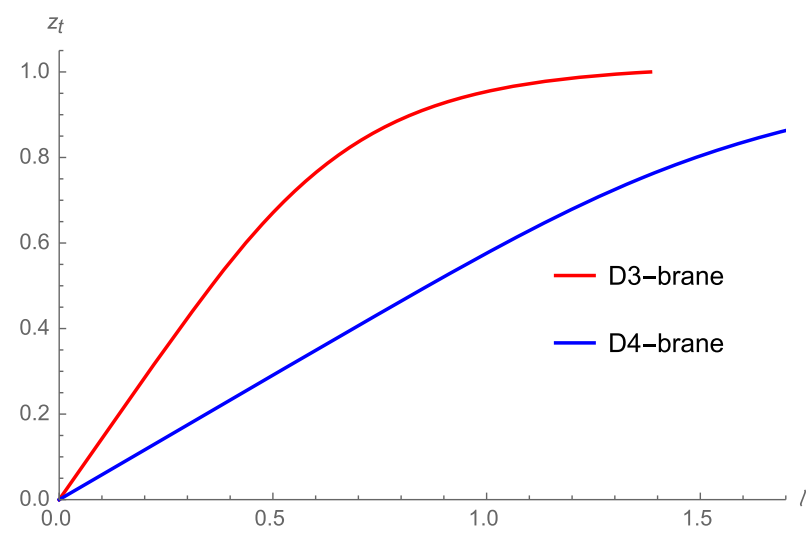

FIG. 2. $\quad z_{t}$ vs $\ell$ plot for different $D p$-brane backgrounds. The turning point vanishes at $\ell=0$, and as $\ell$ increases, $z_{t}$ approaches 1 . Here, we set the horizon radius $z_{0}=1$. 


$$
\begin{aligned}
\mathrm{E}_{W_{\mathrm{LT}}}^{(D 4)}= & \frac{2 L^{3}\left(g_{\mathrm{YM}}^{2} N\right)}{G_{\mathrm{N}}^{6}} \sum_{n=0}^{\infty} \frac{\Gamma\left(n+\frac{1}{2}\right)}{\sqrt{\pi} \Gamma(n+1)} \\
& \times\left(\frac{z_{t}(2 \ell+h)^{6 n-4}-z_{t}(h)^{6 n-4}}{6 n-4}\right) \cdot \frac{1}{z_{0}^{6 n}} \\
= & \frac{2 L^{3}\left(g_{\mathrm{YM}}^{2} N\right)}{G_{\mathrm{N}}^{6}}\left[\frac{1028 \pi^{2} \Gamma\left(\frac{3}{5}\right)^{4}}{\Gamma\left(\frac{1}{10}\right)^{4}}\left(\frac{1}{h^{4}}-\frac{1}{(2 \ell+h)^{4}}\right)\right. \\
& +\frac{25}{16 \pi} \frac{\Gamma\left(\frac{11}{10}\right)^{2}}{\Gamma\left(\frac{3}{5}\right)^{2}}\left(1-\frac{2 \Gamma\left(\frac{11}{10}\right) \Gamma\left(\frac{6}{5}\right)}{\Gamma\left(\frac{3}{5}\right) \Gamma\left(\frac{17}{10}\right)}\right) \ell(\ell+h)\left(\frac{8 \pi T}{3}\right)^{6} \\
& +\cdots] .
\end{aligned}
$$

Also, at high temperature, the corresponding EWCS behaves as

$$
\begin{aligned}
\mathrm{E}_{W_{\mathrm{HT}}}^{(D 4)} \approx & \frac{L^{3}\left(g_{\mathrm{YM}}^{2} N\right)}{4 G_{\mathrm{N}}^{6} \Delta^{3}} T^{4}\left[\tilde{\mathcal{C}}_{4}\left(\frac{8 \pi}{3}\right)^{4}+\frac{1024 \pi^{2} \Gamma\left(\frac{3}{5}\right)^{4}}{\Gamma\left(\frac{1}{10}\right)^{4}}\left(\frac{1}{h T}\right)^{4}\right. \\
& \left.+\frac{25}{32 \pi} \frac{\Gamma\left(\frac{11}{10}\right)^{3} \Gamma\left(\frac{6}{5}\right)}{\Gamma\left(\frac{3}{5}\right)^{3} \Gamma\left(\frac{17}{10}\right)}\left(\frac{8 \pi}{3}\right)^{6}(h T)^{2}\right]
\end{aligned}
$$

where $\tilde{\mathcal{C}}_{4}=\sum_{n=0}^{\infty} \frac{\Gamma\left(n+\frac{1}{2}\right)}{\sqrt{\pi}(6 n-4) \Gamma(n+1)}$.
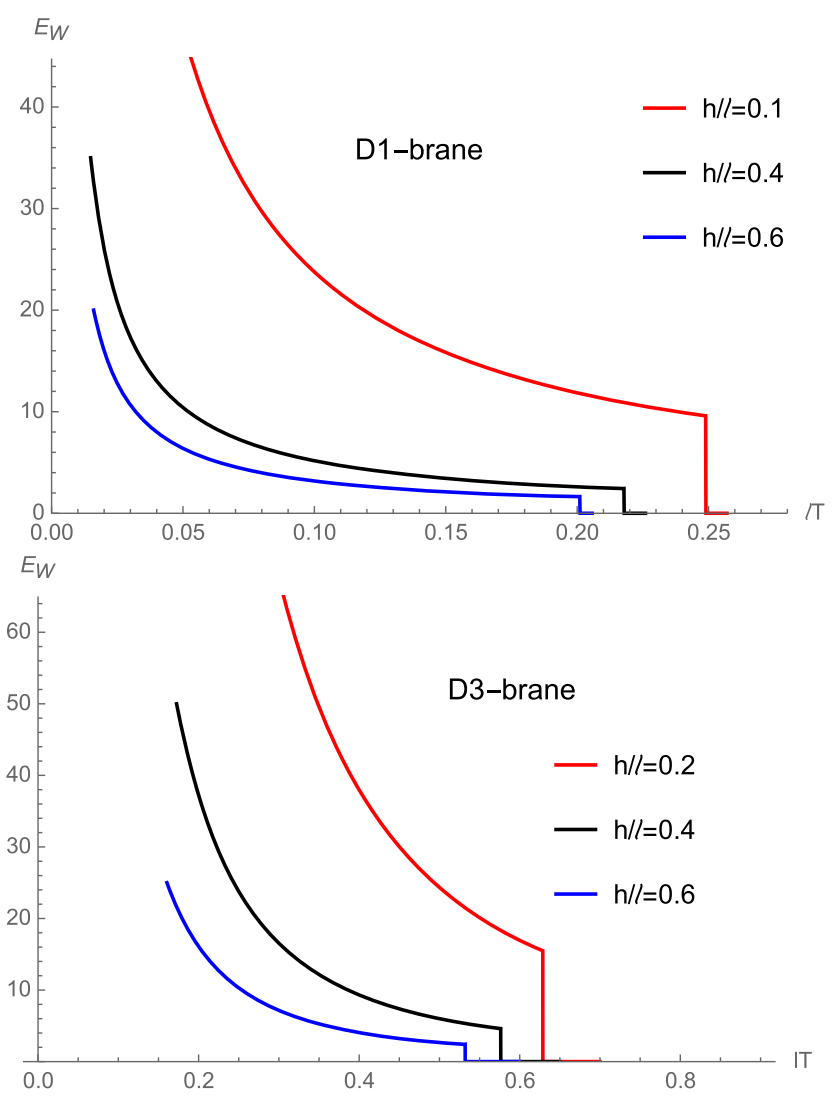

FIG. 3. $\quad \mathrm{E}_{W}$ vs $\ell T$ plots for different values of the dimensionless quantity $h / \ell$. We have set the prefactor $\frac{L^{p-1}}{4 G_{\mathrm{N}}^{p+2}} \frac{\left(g_{\mathrm{YM}}^{2} N\right)^{\frac{(p-3)}{(-p)}}}{\Delta^{\frac{p-1}{5-p}}}=1$. Clearly, as the temperatures $(\ell=1)$ increase, $\mathrm{E}_{W}$ shows a decreasing behavior. In all cases, the $E_{W}$ sharply drops down to zero at some critical value of $\ell$. The behavior of $E_{W}$ corresponding to the conformal D3-brane is also shown.

Notice that we can numerically integrate the integration appearing in (14) for given values of $p$ exactly. In Figs. 3 and 4 , we plot numerically the behavior of $E_{W}$ for different values of the ratio $h / \ell$ for different $D p$-brane backgrounds.

Let us now discuss the following observations that we can make from the low as well as high temperature behaviors of the EWCS derived above. These are listed as follows:

(i) When the widths of the subsystems $(\ell)$ are small, the EWCS increase sharply (Fig. 3). On the other hand, for a given value of the ratio $h / \ell$, it converges to a finite value as $\ell$ increases. Also, beyond certain values of the width, it sharply drops down to zero value, indicating a phase transition due to the disentanglement of the subsystems. For the intermediate values of $\ell$, the decrease in EWCS is monotonic.

(ii) At low temperatures, the first terms of the EWCS in (39), (41), (43), and (45) increase as the separation $h$ between the two entangling regions decreases. Moreover, as $h \rightarrow 0$, the EWCS diverges. This is clearly visible from Fig. 4. Also, if we keep the width and separation between the two subregions fixed and increase the temperature, the EWCS decreases monotonically to zero $\left(\mathrm{E}_{W}=0\right)$ in all cases,
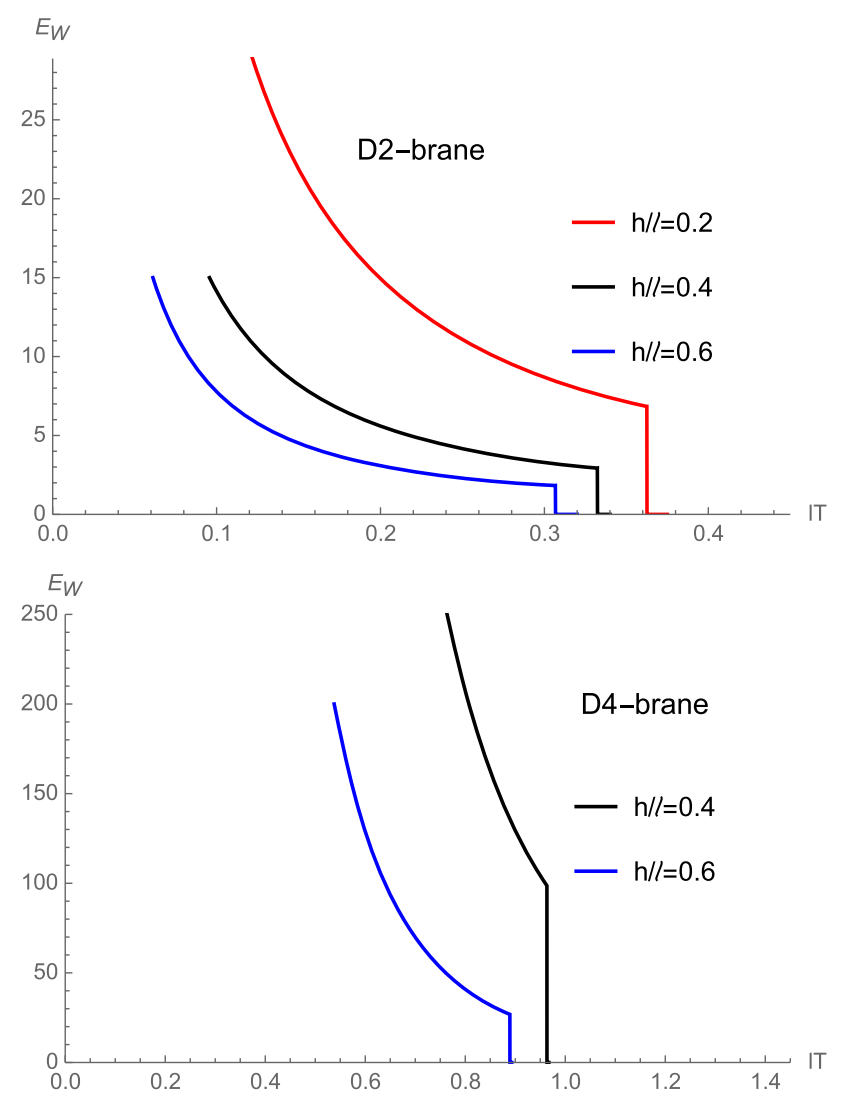

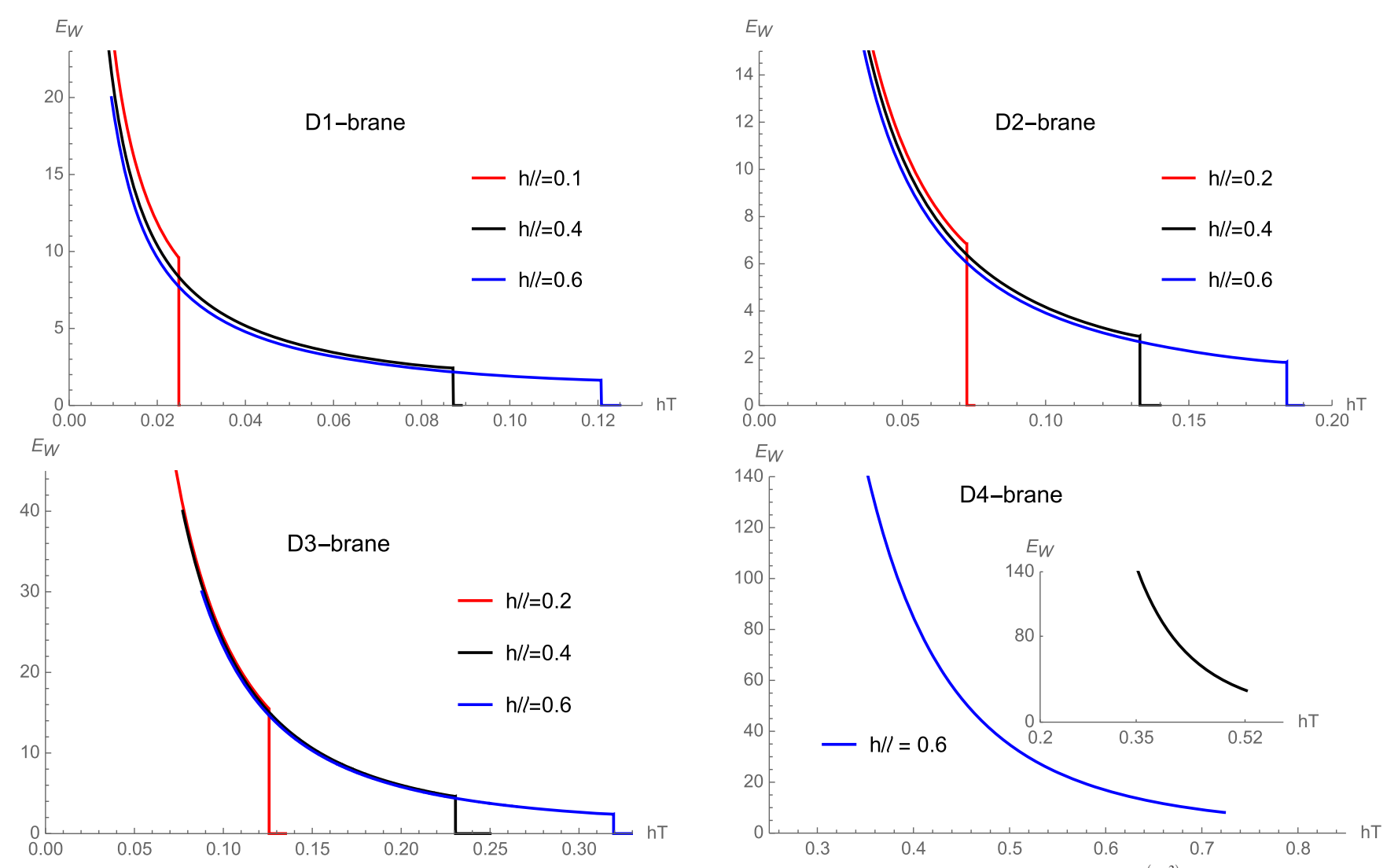

FIG. 4. $\quad \mathrm{E}_{W}$ vs $h T$ plots for different values of the dimensionless quantity $h / \ell$. Here, we have set $\frac{L^{p-1}}{4 G_{\mathrm{N}}^{p+2}} \frac{\left(g_{\mathrm{YM}}^{2} N\right)^{\frac{(p-3)}{(5-p)}}}{\Delta^{\frac{p-1}{5-p}}}=1$. For clarity, the plot corresponding to $h / \ell=0.4$ for the D4-brane is given in the inset. In all cases, the $E_{W}$ jumps discontinuously to zero at some critical value of $h$. The plot corresponding to the conformal D3-brane is also given for comparison.

indicating a phase transition, resulting due to the disentanglement of the Ryu-Takayanagi surfaces.

(iii) The first terms in (40), (42), (44), and (46) are proportional to the area of the entangling region, $L^{p-1}$. This suggests that at finite temperature, the EWCS obeys an area law. This is in sharp contrast to the HEE at high temperature obtained in (37), where it scales with the volume. Similar observations have in fact been made earlier [19-21]. Moreover, this area law scaling implies that the EWCS carries more information than the HEE regarding the correlation between $A$ and $B$ [10-12,19-21].

\section{Critical separation between the strips}

In this section, we determine the critical separation between the parallel strips $A$ and $B$ at which these two subregions become completely disentangled. This can indeed be found by calculating the HMI between $A$ and $B$ [9-12]. The HMI between the two subregions $A$ and $B$ can be expressed as [9-12,19-21]

$$
\mathrm{I}_{M}(\ell, h)=2 S(\ell)-S(h)-S(2 \ell+h) .
$$

It measures the total correlations between $A$ and $B$.
At the critical value of the separation, $h_{c}$, at which the two subregions are no longer entangled, we have

$$
\mathrm{I}_{M}\left(\ell, h_{c}\right)=0 .
$$

This is reminiscent of a first order phase transition, which occurs due to the competition between different configurations for calculating $S(2 \ell+h)$ : When the separation distance is small, the connected configuration $\left(S_{\text {con }}\right)$ is preferred over the disconnected $\left(S_{\mathrm{dis}}\right)$ one. On the other hand, for large separation, the disconnected configuration is preferred, resulting in the vanishing of the HMI $[9,11,19]$. This type of phase transitions occur for large values of the subsystem size $\ell \rightarrow \infty$. Thus, $S(\ell)$ and $S(2 \ell+h)$ are represented by the IR (high temperature) expression for the HEE, while $S(h)$ is represented by the UV (low temperature) expression for the HEE [13,14,19-21].

Before proceeding to the finite temperature analysis, we notice that in the limit $\ell T \rightarrow 0$, i.e., very close to zero temperature, one can calculate the critical values of the ratio $\chi=h / \ell$ from the leading finite terms of (27), (29), (31), and (33). In order to do that, we note that in the disconnected phase, the expression for HEE may be written as [9] 

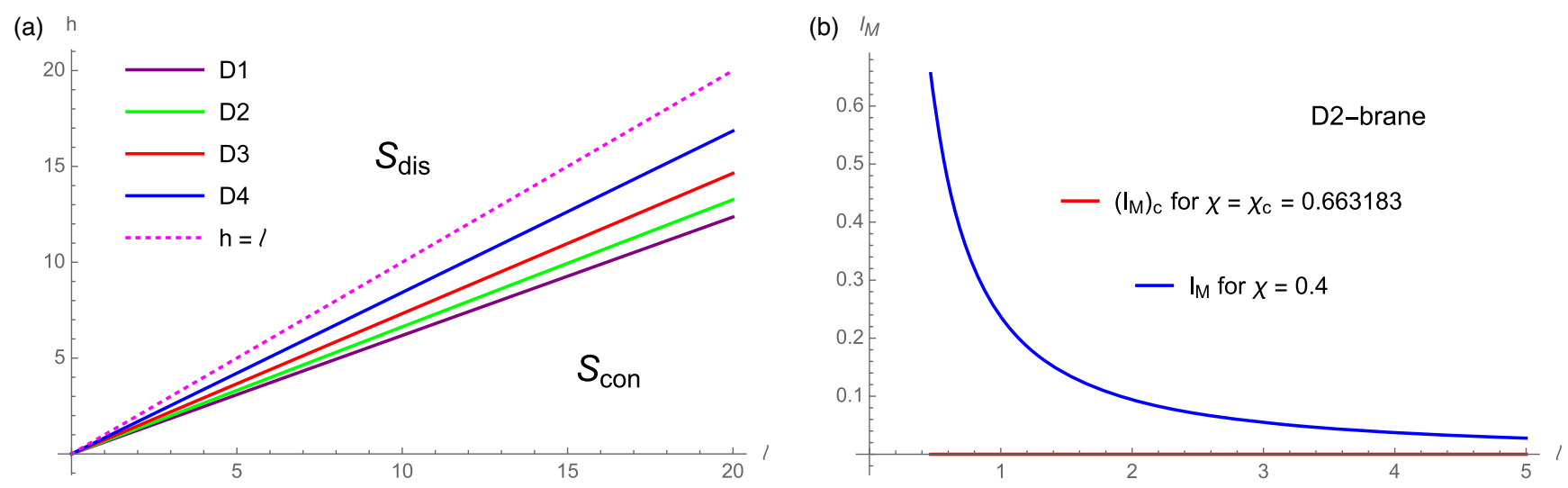

FIG. 5. (a) The phase diagrams corresponding to different $D p$-branes. The entire region above an individual curve corresponds to the disconnected phase $\left(S_{\mathrm{dis}}\right)$, while that below, an individual curve corresponds to the connected phase $\left(S_{\text {con }}\right)$. The dotted line corresponds to $h=\ell$. (b) Mutual information $\left(\mathrm{I}_{M}\right)$ versus strip width $(\ell)$ plot for D2-brane.

$$
S_{\mathrm{dis}}=2 S(\ell)
$$

whereas, for the connected phase,

$$
S_{\mathrm{con}}=S(h)+S(2 \ell+h) .
$$

Now, along the transition lines $\chi_{c}=$ constant, $S_{\text {dis }}=$ $S_{\text {con }}$. This allows us to write the corresponding equations for $\chi_{c}$ as [9]

$$
2= \begin{cases}\frac{1}{\chi_{c}}+\frac{1}{\left(2+\chi_{c}\right)} & \text { for D1-brane, } \\ \frac{1}{\chi_{c}^{4 / 3}}+\frac{1}{\left(2+\chi_{c}\right)^{4 / 3}} & \text { for D2-brane, } \\ \frac{1}{\chi_{c}^{2}}+\frac{1}{\left(2+\chi_{c}\right)^{2}} & \text { for D3-brane, } \\ \frac{1}{\chi_{c}^{4}}+\frac{1}{\left(2+\chi_{c}\right)^{4}} & \text { for D4-brane }\end{cases}
$$

From (51), we can compute the critical values as $\chi_{c}=0.618034,0.663183,0.732051,0.842514$ for D1-, D2-, D3-, D4-brane, respectively. We also observe that, as $p$ increases, the slope of the corresponding critical line also increases.

In Fig. 5(a), we have shown the phase diagrams corresponding to the $D p$-branes. Whereas, in Fig. 5(b), the behavior of the mutual information $\left(I_{M}\right)$ has been shown for D2-brane. Similar plots can be drawn for other backgrounds as well. Notice that, as long as $\chi<\chi_{c}$ $\left(\chi_{c}=0.663183\right.$ for D2-brane [9]), $\mathrm{I}_{M}$ is a monotonically decreasing function of $\ell$; while, at $\chi_{c}$, it vanishes. Similar behaviors of $I_{M}$ were observed for pure $\operatorname{AdS}_{d+1}$ backgrounds in [20]. Also note that, in plotting Fig. 5(b) numerically, we have used (36) and (47).

We now move on to the finite temperature cases and compute the critical separation, $h_{c}$. However, due to the complexity of the resulting expressions that arise from (48), below, we only write down the general form of the equation for the critical separations $\left(h_{c}\right)$ as

$$
\sum_{i=0}^{4} \mathrm{a}_{i}\left(\frac{h_{c}}{z_{0}}\right)^{\mathrm{n}_{i}}=0
$$

where we have used results from subsection A of section III. In (52), the numerical coefficients $\mathrm{a}_{i}$ are certain combinations of gamma functions whose explicit expressions can be written in terms of various constants that appear in subsection A of section III and $\Theta$ in (37) and are presented in appendix. On the other hand, the exponents $\mathrm{n}_{i}$ may be given as ${ }^{9}$

for D1-brane:

$$
\left(\mathrm{n}_{0}=0, \mathrm{n}_{1}=1, \mathrm{n}_{2}=2, \mathrm{n}_{3}=3, \mathrm{n}_{4}=6\right),
$$

for D2-brane:

$$
\left(\mathrm{n}_{0}=0, \mathrm{n}_{1}=\frac{4}{3}, \mathrm{n}_{2}=\frac{7}{3}, \mathrm{n}_{3}=\frac{10}{3}, \mathrm{n}_{4}=\frac{20}{3}\right),
$$

for D3-brane:

$$
\left(\mathrm{n}_{0}=0, \mathrm{n}_{1}=2, \mathrm{n}_{2}=3, \mathrm{n}_{3}=4, \mathrm{n}_{4}=8\right),
$$

for D4-brane:

$$
\left(\mathrm{n}_{0}=0, \mathrm{n}_{1}=4, \mathrm{n}_{2}=5, \mathrm{n}_{3}=6, \mathrm{n}_{4}=12\right) .
$$

Finally, we explicitly check the inequality (1) by numerically plotting both $\mathrm{E}_{W}$ and $\mathrm{I}_{M}(A, B) / 2$ against the dimensionless quantity $h T$ in Fig. 6. Note that, in plotting Fig. 6, we have used (36) and (47). In addition to validate (1), these plots also show the monotonically decreasing nature of both $\mathrm{E}_{W}$ and $\mathrm{I}_{M}(A, B)$. Moreover, beyond the critical separation $h_{c} T(T=1)$ determined by $(52)$, both of them approach zero, which is an essential nature for the phase transition discussed above $[9,11,13-15]$.

\footnotetext{
${ }^{9}$ The corresponding expression for D3-brane may be compared with that already available in the literature; see, e.g., [20,21].
} 

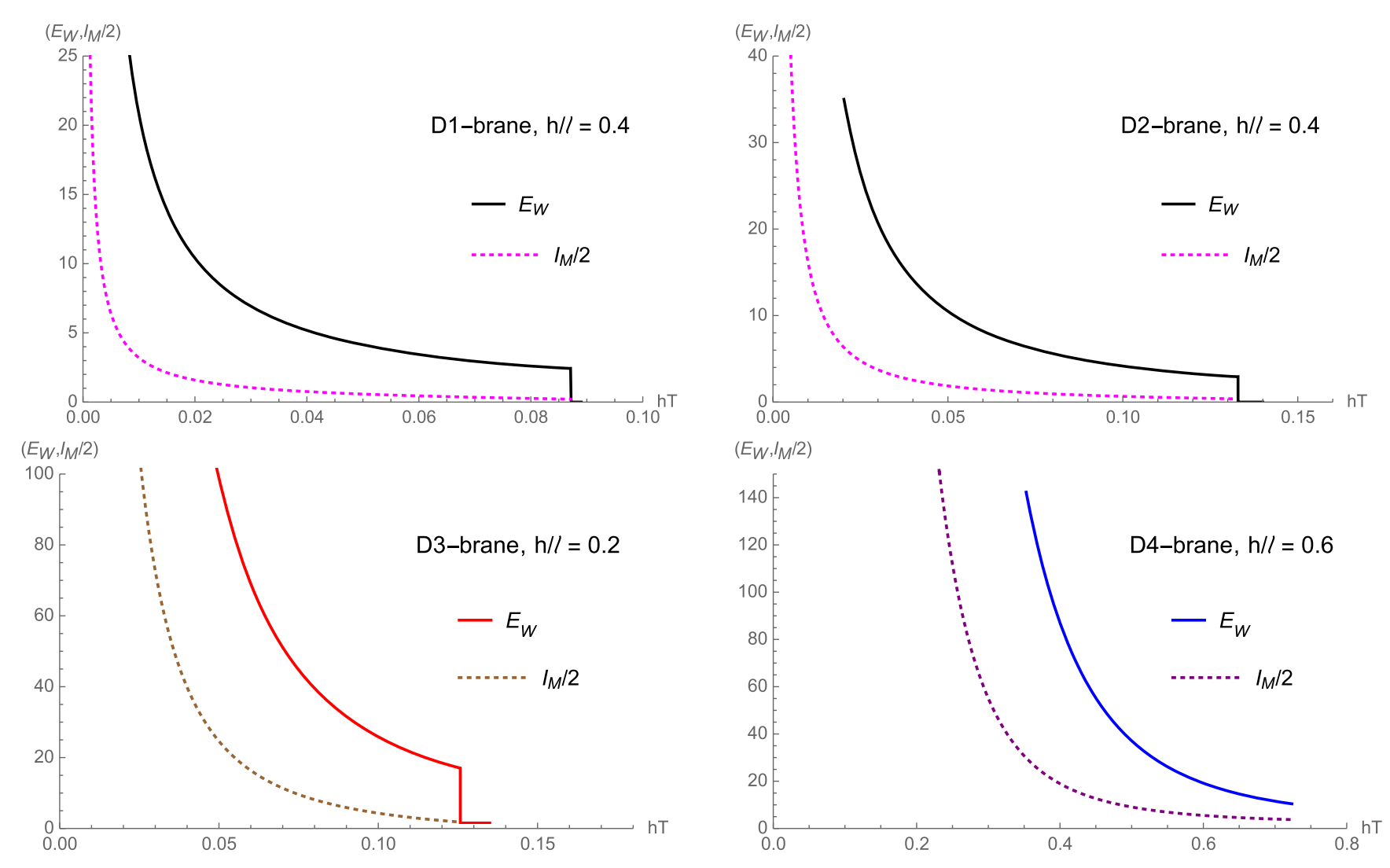

FIG. 6. Holographic mutual information $\left(I_{M} / 2\right)$ plots for different nonconformal backgrounds. Notice that in all the cases, $\mathrm{E}_{W} \geq \mathrm{I}_{M} / 2$. We set $\frac{L^{p-1}}{4 G_{\mathrm{N}}^{p+2}} \frac{\left(g_{\mathrm{YM}}^{2} N\right)^{\frac{(p-3)}{(5-p)}}}{\Delta^{\frac{p-1}{5-p}}}=1$.

\section{CONCLUSIONS}

In this paper, we have studied aspects of holographic entanglement measures for certain class of nonconformal field theories, which are holographic dual to nonconformal $D p$-brane backgrounds [28-32]. Within the framework of the gauge/gravity duality, we explicitly computed EWCS, HEE, and HMI for these field theories. In our analysis, we considered $p=1,2,4$; while $p=3$ corresponds to the usual $\mathrm{AdS}_{5} / \mathrm{CFT}_{4}$ duality [1]. In our computations, we considered a bipartite system with two parallel entangling regions of equal width $\ell$ and length $L$, separated by a distance $h$. Implementing analytical as well as numerical methods, we observe that the qualitative behaviors of the above mentioned holographic measures are similar to those of the conformal case: Both the EWCS and HMI show monotonically decreasing behavior with temperature. However, while the previous one discontinuously drops down to zero value beyond a critical separation distance $h_{c}$ between the entangling subregions, the later attains zero value at $h_{c}$ in a smooth and continuous fashion. This suggests a first order phase transition between connected and disconnected phases of the bipartite system. In our analysis, we have also been able to compute this critical values analytically. Our analysis indeed establishes the fact that the qualitative behaviors of the holographic information quantities are similar irrespective of whether the boundary field theory is conformal or not.

We believe that this nontrivial analysis of information quantities, in the light of gauge/gravity correspondence, will be extremely useful in order to understand aspects of field theories with explicit breaking of conformal invariance. From this point of view, it seems fascinating to consider quantum corrections to both EWCS and HEE in these holographic setups [8]. On top of that, other relevant measures, such as entanglement negativity [16], reflected entropy [17,18], etc., can also be studied within these nonconformal theories. It will be equally interesting to study the evolution and scaling of EWCS after thermal as well as electromagnetic quenches [25-27] corresponding to these theories. These, we plan to explore in future.

\section{ACKNOWLEDGMENTS}

The present work is supported by the Chilean National Agency for Research and Development (ANID)/ FONDECYT/POSTDOCTORADO BECAS CHILE/ Project No. 3190021. A. L. would like to thank 
Shankhadeep Chakrabortty, Suvankar Dutta, Radouane Gannouji, M. Reza Mohammadi Mozaffar, and especially

Karunava Sil for very useful discussions. A. L. would also like to thank the referee for her or his valuable comments.

\section{APPENDIX: VARIOUS COEFFICIENTS} APPEARING IN SUBSECTION A OF SECTION III

The coefficients appearing in (27) [D1-brane] are given by

$$
\begin{aligned}
& \mathcal{C}_{1}=\frac{\pi \Gamma\left(\frac{-1}{4}\right) \Gamma\left(\frac{3}{4}\right)}{16 \Gamma\left(\frac{1}{4}\right) \Gamma\left(\frac{5}{4}\right)}, \\
& \mathcal{C}_{2}=\frac{1}{\mathcal{C}_{1}} \frac{16 \Gamma\left(\frac{5}{4}\right)^{2}}{\Gamma\left(\frac{3}{4}\right)^{2}}\left(1+\frac{\Gamma\left(\frac{-1}{4}\right) \Gamma\left(\frac{5}{4}\right)}{2 \Gamma\left(\frac{1}{4}\right) \Gamma\left(\frac{3}{4}\right)}\right), \\
& \mathcal{C}_{3}=\frac{1}{\mathcal{C}_{1}} \frac{4096 \Gamma\left(\frac{5}{4}\right)^{6}}{\pi \Gamma\left(\frac{3}{4}\right)^{6}}\left[\frac{4 \Gamma\left(\frac{3}{4}\right)}{5 \pi^{3 / 2}}+\frac{3 \Gamma\left(\frac{9}{4}\right) \Gamma\left(\frac{-1}{4}\right)}{2 \pi \Gamma\left(\frac{1}{4}\right) \Gamma\left(\frac{11}{4}\right)}-1\right] .
\end{aligned}
$$

The coefficients appearing in (29) [D2-brane] are written as

$$
\begin{aligned}
\mathcal{D}_{1}= & \frac{2^{8 / 3} \pi^{7 / 6} \Gamma\left(\frac{-2}{7}\right)}{49 \sqrt[3]{21} \Gamma\left(\frac{3}{14}\right)}\left(\frac{\Gamma\left(\frac{5}{7}\right)}{\Gamma\left(\frac{17}{14}\right)}\right)^{4 / 3}, \\
\mathcal{D}_{2}= & \frac{1}{\mathcal{D}_{1}} \frac{63 \Gamma\left(\frac{17}{14}\right)^{3}}{8 \sqrt{\pi} \Gamma\left(\frac{5}{7}\right)^{3}}\left[\frac{\Gamma\left(\frac{-2}{7}\right) \Gamma\left(\frac{10}{7}\right)}{\Gamma\left(\frac{3}{14}\right) \Gamma\left(\frac{27}{14}\right)}+\frac{3 \Gamma\left(\frac{3}{7}\right) \Gamma\left(\frac{5}{7}\right)}{\Gamma\left(\frac{17}{14}\right) \Gamma\left(\frac{13}{4}\right)}\right], \\
\mathcal{D}_{3}= & \frac{1}{\mathcal{D}_{1}}\left[-\frac{1750329 \sqrt[3]{21} \Gamma\left(\frac{3}{7}\right) \Gamma\left(\frac{17}{14}\right)^{19 / 3} \Gamma\left(\frac{10}{7}\right)}{20482^{2 / 3} \pi^{13 / 6} \Gamma\left(\frac{5}{7}\right)^{19 / 3} \Gamma\left(\frac{13}{14}\right) \Gamma\left(\frac{27}{14}\right)}\right. \\
& +\frac{2^{2 / 3} \pi^{7 / 6} \Gamma\left(\frac{-2}{7}\right)}{245 \sqrt[3]{21} \Gamma\left(\frac{3}{14}\right)}\left(\frac{\Gamma\left(\frac{5}{7}\right)}{\Gamma\left(\frac{17}{14}\right)}\right)^{4 / 3}\left(\frac{33353491521^{2 / 3} \Gamma\left(\frac{17}{14}\right)^{26 / 3} \Gamma\left(\frac{10}{7}\right)^{2}}{4096 \sqrt[3]{2} \pi^{10 / 3} \Gamma\left(\frac{5}{7}\right)^{26 / 3} \Gamma\left(\frac{27}{14}\right)^{2}}\right. \\
& \left.+\frac{14294353521^{2 / 3} \Gamma\left(\frac{17}{14}\right)^{20 / 3}\left(3 \Gamma\left(\frac{5}{7}\right) \Gamma\left(\frac{17}{14}\right) \Gamma\left(\frac{27}{14}\right)^{2} \Gamma\left(\frac{15}{7}\right)-2 \Gamma\left(\frac{17}{14}\right)^{2} \Gamma\left(\frac{10}{7}\right)^{2} \Gamma\left(\frac{37}{14}\right)\right)}{4096 \sqrt[3]{2} \pi^{10 / 3} \Gamma\left(\frac{5}{7}\right)^{26 / 3} \Gamma\left(\frac{27}{14}\right)^{2} \Gamma\left(\frac{37}{14}\right)}\right) \\
& +\frac{5250987 \sqrt[3]{21} \Gamma\left(\frac{8}{7}\right) \Gamma\left(\frac{17}{14}\right)^{16 / 3}}{81922^{2 / 3} \pi^{13 / 6} \Gamma\left(\frac{5}{7}\right)^{16 / 3} \Gamma\left(\frac{23}{7}\right)} .
\end{aligned}
$$

The coefficients in (31) [D3-brane] are of the following forms:

$$
\begin{aligned}
\mathcal{B}_{1}= & \frac{\pi^{3 / 2} \Gamma\left(\frac{-1}{3}\right) \Gamma\left(\frac{2}{3}\right)^{2}}{27 \Gamma\left(\frac{1}{6}\right) \Gamma\left(\frac{7}{6}\right)^{2}}, \\
\mathcal{B}_{2}= & \frac{1}{\mathcal{B}_{1}}\left(\frac{3 \Gamma\left(\frac{4}{3}\right) \Gamma\left(-\frac{1}{3}\right) \Gamma\left(\frac{7}{6}\right)^{3}}{\sqrt{\pi} \Gamma\left(\frac{1}{6}\right) \Gamma\left(\frac{2}{3}\right)^{3} \Gamma\left(\frac{11}{6}\right)}+\frac{3 \Gamma\left(\frac{1}{3}\right) \Gamma\left(\frac{7}{6}\right)^{2}}{2 \sqrt{\pi} \Gamma\left(\frac{2}{3}\right)^{2} \Gamma\left(\frac{5}{6}\right)}\right), \\
\mathcal{B}_{3}= & \frac{1}{\mathcal{B}_{1}}\left[\frac{243 \Gamma\left(\frac{7}{6}\right)^{7}}{2 \pi^{5 / 2} \Gamma\left(\frac{2}{3}\right)^{7}}\left(\frac{3 \Gamma\left(\frac{2}{3}\right)}{8 \Gamma\left(\frac{7}{6}\right)}-\frac{\Gamma\left(\frac{1}{3}\right) \Gamma\left(\frac{4}{3}\right)}{\Gamma\left(\frac{5}{6}\right) \Gamma\left(\frac{11}{6}\right)}\right)\right. \\
& \left.+\frac{\pi^{3 / 2} \Gamma\left(-\frac{1}{3}\right) \Gamma\left(\frac{2}{3}\right)^{2}\left(\frac{\left.39366 \Gamma \Gamma \frac{7}{6}\right)^{10} \Gamma\left(\frac{4}{3}\right)^{2}}{\pi^{4} \Gamma\left(\frac{2}{3}\right)^{10} \Gamma\left(\frac{11}{6}\right)^{2}}-\frac{26244 \Gamma\left(\frac{7}{6}\right)^{9}\left(\sqrt{\pi} \Gamma\left(\frac{7}{6}\right) \Gamma\left(\frac{4}{3}\right)^{2}-2 \Gamma\left(\frac{2}{3}\right)^{2}\left(\frac{11}{6}\right)^{2}\right)}{\pi^{9 / 2} \Gamma\left(\frac{2}{3}\right)^{10} \Gamma\left(\frac{11}{6}\right)^{2}}\right)}{216 \Gamma\left(\frac{1}{6}\right) \Gamma\left(\frac{7}{6}\right)^{2}}\right] .
\end{aligned}
$$

TABLE I. Values of the coefficients $\mathrm{a}_{i}$ in (52) corresponding to different background configurations. Here, $\Theta_{p=\#}$ corresponds to the value of the coefficient $\Theta$ in (37) for different values of $p(=1,2,3,4)$.

\begin{tabular}{llllll}
\hline \hline Background & $\mathrm{a}_{0}$ & $\mathrm{a}_{1}$ & $\mathrm{a}_{2}$ & $\mathrm{a}_{3}$ & $\mathrm{a}_{4}$ \\
\hline D1 & $\mathcal{C}_{1}$ & $-\Theta_{p=1} / 2$ & 2 & $\mathcal{C}_{1} \mathcal{C}_{2}$ & $\mathcal{C}_{1} \mathcal{C}_{3}$ \\
D2 & $\mathcal{D}_{1}$ & $-\Theta_{p=2} / 2$ & $9 / 8$ & $\mathcal{D}_{1} \mathcal{D}_{2}$ & $\mathcal{D}_{1} \mathcal{D}_{3}$ \\
D3 & $\mathcal{B}_{1}$ & $-\Theta_{p=3} / 2$ & $1 / 2$ & $\mathcal{B}_{1} \mathcal{B}_{2}$ & $\mathcal{B}_{1} \mathcal{B}_{3}$ \\
D4 & $\mathcal{E}_{1}$ & $-\Theta_{p=4} / 2$ & $1 / 8$ & $\mathcal{E}_{1} \mathcal{E}_{2}$ & $\mathcal{E}_{1} \mathcal{E}_{3}$ \\
\hline \hline
\end{tabular}


Finally, the coefficients in (33) [D4-brane] are written as

$$
\begin{aligned}
\mathcal{E}_{1}= & \frac{256 \pi^{5 / 2} \Gamma\left(\frac{-2}{5}\right) \Gamma\left(\frac{3}{5}\right)^{4}}{3125 \Gamma\left(\frac{1}{10}\right) \Gamma\left(\frac{11}{10}\right)^{4}}, \\
\mathcal{E}_{2}= & \frac{1}{\mathcal{E}_{1}} \frac{5 \Gamma\left(\frac{11}{10}\right)^{3}}{8 \sqrt{\pi} \Gamma\left(\frac{3}{5}\right)^{3}}\left(\frac{\Gamma\left(\frac{-2}{5}\right) \Gamma\left(\frac{6}{5}\right)}{\Gamma\left(\frac{1}{10}\right) \Gamma\left(\frac{17}{10}\right)}+\frac{\Gamma\left(\frac{1}{5}\right) \Gamma\left(\frac{3}{5}\right)}{4 \Gamma\left(\frac{7}{10}\right) \Gamma\left(\frac{11}{10}\right)}\right), \\
\mathcal{E}_{3}= & \frac{1}{\mathcal{E}_{1}}\left[-\frac{78125 \Gamma\left(\frac{1}{5}\right) \Gamma\left(\frac{11}{10}\right)^{9} \Gamma\left(\frac{6}{5}\right)}{131072 \pi^{7 / 2} \Gamma\left(\frac{3}{5}\right)^{9} \Gamma\left(\frac{7}{10}\right) \Gamma\left(\frac{17}{10}\right)}+\frac{234375 \Gamma\left(\frac{4}{5}\right) \Gamma\left(\frac{11}{10}\right)^{8}}{524288 \pi^{7 / 2} \Gamma\left(\frac{3}{5}\right)^{8} \Gamma\left(\frac{13}{5}\right)}\right. \\
& +\frac{64 \pi^{5 / 2} \Gamma\left(-\frac{2}{5}\right) \Gamma\left(\frac{3}{5}\right)^{4}\left(\frac{3662109375 \Gamma\left(\frac{6}{5}\right)^{2} \Gamma\left(\frac{11}{10}\right)^{14}}{8388608 \pi^{6} \Gamma\left(\frac{3}{5}\right)^{14} \Gamma\left(\frac{17}{10}\right)^{2}}+\frac{732421875\left(3 \Gamma\left(\frac{3}{5}\right) \Gamma\left(\frac{17}{10}\right)^{2} \Gamma\left(\frac{9}{5}\right)-2 \Gamma\left(\frac{11}{10}\right) \Gamma\left(\frac{6}{5}\right)^{2} \Gamma\left(\frac{23}{10}\right) \Gamma\left(\frac{11}{10}\right)^{13}\right.}{8388608 \pi^{6} \Gamma\left(\frac{3}{5}\right)^{14} \Gamma\left(\frac{17}{10}\right)^{2} \Gamma\left(\frac{23}{10}\right)}\right)}{9375 \Gamma\left(\frac{1}{10}\right) \Gamma\left(\frac{11}{10}\right)^{4}} .
\end{aligned}
$$

The coefficients $a_{i}$ in (52) can be expressed in terms of the constants appearing in (A1)-(A4) as follows in Table I:

[1] J. M. Maldacena, The Large $N$ limit of superconformal field theories and supergravity, Int. J. Theor. Phys. 38, 1113 (1999); Adv. Theor. Math. Phys. 2, 231 (1998).

[2] E. Witten, Anti-de Sitter space and holography, Adv. Theor. Math. Phys. 2, 253 (1998).

[3] O. Aharony, S. S. Gubser, J. M. Maldacena, H. Ooguri, and Y. Oz, Large $N$ field theories, string theory and gravity, Phys. Rep. 323, 183 (2000).

[4] S. Ryu and T. Takayanagi, Holographic Derivation of Entanglement Entropy from AdS/CFT, Phys. Rev. Lett. 96, 181602 (2006).

[5] S. Ryu and T. Takayanagi, Aspects of holographic entanglement entropy, J. High Energy Phys. 08 (2006) 045 .

[6] V.E. Hubeny, M. Rangamani, and T. Takayanagi, A Covariant holographic entanglement entropy proposal, J. High Energy Phys. 07 (2007) 062.

[7] H. Casini, M. Huerta, and R. C. Myers, Towards a derivation of holographic entanglement entropy, J. High Energy Phys. 05 (2011) 036.

[8] T. Faulkner, A. Lewkowycz, and J. Maldacena, Quantum corrections to holographic entanglement entropy, J. High Energy Phys. 11 (2013) 074.

[9] O. Ben-Ami, D. Carmi, and J. Sonnenschein, Holographic entanglement entropy of multiple strips, J. High Energy Phys. 11 (2014) 144.

[10] M. M. Wolf, F. Verstraete, M. B. Hastings, and J. I. Cirac, Area Laws in Quantum Systems: Mutual Information and Correlations, Phys. Rev. Lett. 100, 070502 (2008).

[11] M. Headrick, Entanglement Renyi entropies in holographic theories, Phys. Rev. D 82, 126010 (2010).

[12] W. Fischler, A. Kundu, and S. Kundu, Holographic mutual information at finite temperature, Phys. Rev. D 87, 126012 (2013).

[13] T. Takayanagi and K. Umemoto, Entanglement of purification through holographic duality, Nat. Phys. 14, 573 (2018).
[14] P. Nguyen, T. Devakul, M. G. Halbasch, M. P. Zaletel, and B. Swingle, Entanglement of purification: From spin chains to holography, J. High Energy Phys. 01 (2018) 098.

[15] B. M. Terhal, M. Horodecki, D. W. Leung, and D. P. DiVincenzo, The entanglement of purification, J. Math. Phys. (N.Y.) 43, 4286 (2002).

[16] J. Kudler-Flam and S. Ryu, Entanglement negativity and minimal entanglement wedge cross sections in holographic theories, Phys. Rev. D 99, 106014 (2019).

[17] S. Dutta and T. Faulkner, A canonical purification for the entanglement wedge cross-section, arXiv:1905.00577.

[18] H. S. Jeong, K. Y. Kim, and M. Nishida, Reflected entropy and entanglement wedge cross section with the first order correction, J. High Energy Phys. 12 (2019) 170.

[19] K. Babaei Velni, M. R. Mohammadi Mozaffar, and M. H. Vahidinia, Some aspects of entanglement wedge crosssection, J. High Energy Phys. 05 (2019) 200.

[20] N. Jokela and A. Pönni, Notes on entanglement wedge cross sections, J. High Energy Phys. 07 (2019) 087.

[21] S. Chakrabortty, S. Pant, and K. Sil, Effect of back reaction on entanglement and subregion volume complexity in strongly coupled plasma, J. High Energy Phys. 06 (2020) 061.

[22] Y. F. Huang, Z. J. Shi, C. Niu, C. Y. Zhang, and P. Liu, Mixed state entanglement for holographic axion model, Eur. Phys. J. C 80, 426 (2020).

[23] J. Boruch, Entanglement wedge cross-section in shock wave geometries, J. High Energy Phys. 07 (2020) 208.

[24] G. Fu, P. Liu, H. Gong, X. M. Kuang, and J. P. Wu, Informational properties for Einstein-Maxwell-Dilaton gravity, arXiv:2007.06001.

[25] R. Q. Yang, C. Y. Zhang, and W. M. Li, Holographic entanglement of purification for thermofield double states and thermal quench, J. High Energy Phys. 01 (2019) 114.

[26] J. Kudler-Flam, Y. Kusuki, and S. Ryu, Correlation measures and the entanglement wedge cross-section after quantum quenches in two-dimensional conformal field theories, J. High Energy Phys. 04 (2020) 074. 
[27] K. Babaei Velni, M. R. Mohammadi Mozaffar, and M. H. Vahidinia, Evolution of entanglement wedge cross section following a global quench, J. High Energy Phys. 08 (2020) 129.

[28] N. Itzhaki, J. M. Maldacena, J. Sonnenschein, and S. Yankielowicz, Supergravity and the large $N$ limit of theories with sixteen supercharges, Phys. Rev. D 58, 046004 (1998).

[29] H. J. Boonstra, K. Skenderis, and P. K. Townsend, The domain wall / QFT correspondence, J. High Energy Phys. 01 (1999) 003; K. Skenderis, Field theory limit of branes and gauged supergravities, Fortschr. Phys. 48, 205 (2000).

[30] I. Kanitscheider, K. Skenderis, and M. Taylor, Precision holography for nonconformal branes, J. High Energy Phys. 09 (2008) 094.

[31] D. W. Pang, Entanglement thermodynamics for nonconformal D-branes, Phys. Rev. D 88, 126001 (2013).

[32] D. W. Pang, Corner contributions to holographic entanglement entropy in nonconformal backgrounds, J. High Energy Phys. 09 (2015) 133.
[33] K. Narayan, On Lifshitz scaling and hyperscaling violation in string theory, Phys. Rev. D 85, 106006 (2012).

[34] H. Singh, Lifshitz/Schrödinger Dp-branes and dynamical exponents, J. High Energy Phys. 07 (2012) 082.

[35] K. Narayan, nonconformal brane plane waves and entanglement entropy, Phys. Lett. B 726, 370 (2013).

[36] R. Mishra and H. Singh, Entanglement asymmetry for boosted black branes and the bound, Int. J. Mod. Phys. A 32, 1750091 (2017).

[37] A. van Niekerk, Entanglement entropy in nonconformal holographic theories, arXiv:1108.2294.

[38] W. Fischler and S. Kundu, Strongly coupled gauge theories: High and low temperature behavior of non-local observables, J. High Energy Phys. 05 (2013) 098.

[39] S. Kundu and J. F. Pedraza, Aspects of holographic entanglement at finite temperature and chemical potential, J. High Energy Phys. 08 (2016) 177.

[40] R. M. Wald, General Relativity (Chicago University Press, Chicago, IL, 1984). 